\title{
STUDY OF THE BILATERAL ASYMMETRY OF PLANTAR MECHANICAL PROPERTIES AS A BIOMARKER FOR THE DIFFERENTIATION OF DIABETIC CONDITION
}

\author{
Shib Sundar Banerjee $^{1}$, Srivatsa Ananthan ${ }^{2}$, and Ramakrishnan Swaminathan ${ }^{1}$ \\ ${ }^{1}$ Department of Applied Mechanics, Indian Institute of Technology Madras, Chennai, India \\ ${ }^{2}$ Sree Clinic and Diabetic Centre, Chennai, India
}

Corresponding Author: Shib Sundar Banerjee

Tel: +9104422575065

Email: banerjeeshib2@gmail.com

https://doi.org/10.34107/BiomedSciInstrum.57.04114

\begin{abstract}
Diabetes mellitus is a globally prevalent metabolic disease which results in altered plantar mechanical properties and foot ulcer. In this study, the bilateral asymmetry of mechanical properties for plantar soft tissue is investigated in healthy and diabetic conditions. Myotonometric signals are acquired from sub-metatarsal region of the plantar faces of healthy subjects and patients with varied diabetic age. Mechanical parameters such as dynamic stiffness and logarithmic decrement are extracted from the recorded signal. The asymmetry indices between right and left feet are computed. Statistical analysis shows that the spatial pattern of dynamic stiffness and logarithmic decrement varies significantly between healthy and diabetic subjects. The asymmetry index of dynamic stiffness in the fifth sub-metatarsal head can differentiate between healthy subjects and patients with both high and low diabetic age $(\mathrm{p}<0.05)$. The asymmetry index of logarithmic decrement is found to vary significantly between the healthy subjects and patients with higher diabetic age $(p<0.05)$. These results indicate that bilateral asymmetry of myotonometric parameters can be exploited as a possible biomarker to differentiate diabetic patients from healthy subjects and can aid in the early detection of foot ulcer.
\end{abstract}

Keywords: Diabetes Mellitus, Foot Ulcer, Plantar Soft Tissue, Myotonometry, Asymmetry Index

\section{INTRODUCTION}

Type 2 Diabetes mellitus is a class of chronic metabolic disease characterized by hyperglycemia and insulin resistance [1]. This disease has become globally prevalent as a fast growing pathology, especially in the south Asian countries [2]. A common manifestation of diabetes is microvascular complications in lower limb and foot ulcer [3]. The management of diabetic lesions in feet is not fool-proof and can eventually require amputation [4]. Early detection of ulceration in feet is clinically relevant as it can help in better diagnosis and management of the diabetic complications [5]. Changes in viscoelastic properties of plantar tissues in diabetic conditions have been reported in earlier studies. Zheng et al [6] reported heightened plantar stiffness in case of diabetic patients as compared to healthy controls. This phenomenon has been attributed to factors like thickening of plantar fascia and loss of soft tissue cover [7]. A foot with less compliance experiences higher ground impacts during gait cycles and it eventually leads to damaged soft tissue and breaking down of skin. Degradation of plantar structure initiates the process of ulceration [8]. Also, the bilateral asymmetry in peak plantar pressure in the metatarsal region has been correlated with prognosis of diabetes and the resulting asymmetric limb function [9], [10]. Altered tissue structure and mechanical characteristics due to diabetes is suggested to have contributed to the pressure asymmetry [11].

Different techniques have been utilized to estimate the viscoelastic properties of plantar. Manual palpation is performed by physicians for foot assessment. Superficial changes in the tissue structure such as induration or calcification is detectable in this method, but it suffers from subjective variability [12]. 